\title{
THE FIXED POINT PROPERTY IN BANACH SPACES WHOSE CHARACTERISTIC OF UNIFORM CONVEXITY IS LESS THAN 2
}

\author{
J. GARCIA FALSET
}

(Received 17 May 1991)

Communicated by P. G. Dodds

\begin{abstract}
We prove that every Banach space $X$ with characteristic of uniform convexity less than 2 has the fixed point property whenever $X$ satisfies a certain orthogonality condition.

1991 Mathematics subject classification (Amer. Math. Soc.): Primary 47 H 10; Secondary 46 B 30.
\end{abstract}

\section{Introduction}

If $B$ is a subset of a Banach space $X$, a map $T: B \rightarrow B$ is said to be a nonexpansive mapping when the inequality $\|T x-T y\| \leq\|x-y\|$ holds for every $x, y \in B$. We say that $X$ has the (weak) fixed point property (FPP) if every nonexpansive mapping on a nonempty weakly compact convex subset of $X$ has a fixed point.

It is known that $L_{1}$ fails to have the FPP [1], but B. Maurey [7] has proved that all reflexive subspaces of $L_{1}$ and also $c_{0}$ have the FPP. In order to generalize these results Borwein and Sims [2] introduced the notion of weak orthogonality in Banach lattices. Later Sims [8] proved that every weakly orthogonal Banach lattice has the FPP and in this paper, he also introduces the notion of weak orthogonality in Banach spaces (WOTH) which is a generalization of weak orthogonality in Banach lattices. Sims also proved that the (WOTH) is related to the non-strict Opial condition, but it is not known

(C) 1993 Australian Mathematical Society 0263-6115/93 \$A2.00+0.00 
whether spaces with WOTH enjoy the FPP.

An open question in fixed point theory is the following. If $X$ is a superreflexive Banach space, does $X$ have the FPP? In particular, if $X$ is a Banach space with $\varepsilon_{0}(X)<2$ (characteristic of uniform convexity), does $X$ have the FPP? (See [3].)

On the other hand, we introduce in [4] a geometric property (AMCP) which is a sufficient condition for the FPP.

In this note, we prove that every Banach space (WOTH) so that $\varepsilon_{0}(X)<2$ has the AMCP and hence the FPP.

\section{Basic definitions and results}

Let $X$ be a Banach space. We denote by $[X]$ the quotient space $l_{\infty}(X) / c_{0}(X)$ endowed with the norm given by $\left|\left[z_{n}\right]\right|=\lim \sup _{n}\left\|z_{n}\right\|$ where $\left[z_{n}\right]$ denotes the equivalence class of $\left(z_{n}\right)+c_{0}(X)$. If $K$ is a nonempty bounded subset of $X$, and $x$ is a point of $X$ we denote by $R(x, K)$ the number: $R(x, K):=\sup \{\|x-z\|: z \in K\}$. When $K$ is also convex, and $x, y$ are points in $K$, the set of quasi-midpoints of $x$ and $y$ in $K$ is:

$$
M(x, y):=\left\{z \in K: \max \{\|z-x\|\|z-y\|\} \leq \frac{1}{2}\|x-y\|\right\} .
$$

We write $[K]:=\left\{\left[z_{n}\right] \in[X]: z_{n} \in K, n=1,2, \ldots\right\}$.

If $K$ is a closed, bounded and convex subset of $X$, then $[K]$ is a closed, bounded and convex subset of $[X]$.

Let $\mathscr{S}(\mathbb{N})$ be the set of all strictly increasing sequences of natural numbers. If $\beta, \gamma$ belong to $\mathscr{S}(\mathbb{N})$ we write $\beta \neq \gamma$ whenever $\beta(n) \neq \gamma(n), n=$ $1,2, \ldots$.

Definition 1.1. Let $M$ be a nonempty bounded convex subset of a $\mathrm{Ba}$ nach space $X$. A sequence $\left(x_{n}\right)$ in $M$ is said to be equilateral in $M$ if for any $\beta, \gamma \in \mathscr{S}(\mathbb{N})$ such that $\beta \neq \gamma$ the following equality holds:

$$
\mid\left[x_{\beta(n)}|=|\left[x_{\beta(n)}\right]-\left[x_{\gamma(n)}\right]|=|\left[x_{\gamma(n)}\right] \mid=D\left(x_{n}\right)=\operatorname{diam}(M)\right.
$$

where $D\left(x_{n}\right):=\lim \sup _{n}\left(\lim \sup _{m}\left\|x_{n}-x_{m}\right\|\right)$ is the asymptotic diameter of $\left(x_{n}\right)$.

Definition 1.2. A bounded closed convex subset $M$ of a Banach space $X$, with $0 \in M$ is said to have the AMC-property if for every weakly null sequence $\left(x_{n}\right)$ which is equilateral in $M$ there exist $\left.\rho \in\right] 0,1[$, and $\beta, \gamma \in$ $\mathscr{S}(\mathbb{N})$ with $\beta \neq \gamma$, such that the set

$$
M_{\rho}\left(\left(x_{n}\right), \beta, \gamma\right):=M\left(\left[x_{\beta(n)}\right],\left[x_{\gamma(n)}\right]\right) \cap\left\{\left[z_{n}\right]: d\left(\left[z_{n}\right], M\right) \leq \rho \operatorname{diam}(M)\right\}
$$

is nonempty and $R\left(x, M_{\rho}\left(\left(x_{n}\right), \beta, \gamma\right)\right)<\operatorname{diam}(M)$ for some $x$ in $M$. 
When every weakly compact convex of $X$ in the above conditions has the AMC-property we say that $X$ has the AMC-property.

Lemma 1.3. Let $X$ be a Banach space. If $X$ has the AMC-property then $X$ has the FPP. (See [4].)

As in [7] a Banach space $X$ is said to have the WOTH if every weakly null sequence $\left(x_{n}\right)$ of $X$ satisfies:

$$
\lim _{n \rightarrow \infty}\left|\left\|x_{n}+x\right\|-\left\|x_{n}-x\right\|\right|=0 \text { for any } x \in X .
$$

If $X$ is a Banach space, we write, as is usual, $\varepsilon_{0}(X):=\sup \{\varepsilon: \delta(\varepsilon)=0\}$ where $\delta()$ is the modulus of convexity of $X$.

\section{The main result}

THEOREM 2.1. Let $X$ be a Banach space so that $\varepsilon_{0}(X)<2$ and moreover $X$ is WOTH then $X$ has the AMCP.

Proof. Let $K$ be a nonempty weakly compact convex subset of $X$ such that $0 \in K$. Without loss of generality, we can assume that $\operatorname{diam}(K)=1$.

Let us see that $K$ has the AMC-property. Indeed, let $\left(x_{n}\right)$ be a weakly null sequence equilateral in $K$. Since $X$ is WOTH we can find $\beta, \gamma \in \mathscr{S}(\mathbb{N})$ with $\beta \neq \gamma$ such that:

$$
\lim _{n \rightarrow \infty}\left|\left\|x_{\beta(n)}+x_{\gamma(n)}\right\|-\left\|x_{\beta(n)}-x_{\gamma(n)}\right\|\right|=0 .
$$

Consequently $\left|\left[x_{\beta(n)}\right]-\left[x_{\gamma(n)}\right]\right|=\left|\left[x_{\beta(n)}\right]+\left[x_{\gamma(n)}\right]\right|$ and since $\left(x_{n}\right)$ is equilateral in $K$, it is known that $\left|\left[x_{\beta(n)}\right]-\left[x_{\gamma(n)}\right]\right|=\operatorname{diam}(K)=1$. Hence $\left|\left[x_{\beta(n)}\right]+\left[x_{\gamma(n)}\right] / 2\right|=1 / 2$.

Consider the set $M_{1 / 2}\left(\left(x_{n}\right), \beta, \gamma\right)$. This set is nonempty since

$$
\frac{\left[x_{\beta(n)}\right]+\left[x_{\gamma(n)}\right]}{2} \in M_{1 / 2}\left(\left(x_{n}\right), \beta, \gamma\right) .
$$

Moreover if $\left[z_{n}\right] \in M_{1 / 2}\left(\left(x_{n}\right), \beta, \gamma\right)$ we know that

$$
\left|\left[z_{n}\right]-\left[x_{\beta(n)}\right]\right| \leq \frac{1}{2} \text { and }\left|\left[z_{n}\right]-\left[x_{\gamma(n)}\right]\right| \leq \frac{1}{2} \text {. }
$$

Hence using the definition of the quotient norm of $[X]$, we deduce that given $\varepsilon>0$ there exists $n_{0} \in \mathbb{N}$ such that for any $n \geq n_{0}$

$$
\begin{gathered}
\left\|z_{n}-x_{\beta(n)}\right\| \leq \frac{1}{2}+\varepsilon, \quad\left\|z_{n}-z_{\beta(n)}\right\| \leq \frac{1}{2}+\varepsilon \quad \text { and } \\
\left\|x_{\beta(n)}-x_{\gamma(n)}\right\| \geq\left(\frac{1}{2}+\varepsilon\right) 2 \frac{1-\varepsilon}{1+2 \varepsilon} .
\end{gathered}
$$


And then for every $n \geq n_{0}$

$$
\left|z_{n}-\frac{x_{\beta(n)}+x_{\gamma(n)}}{2}\right| \leq\left(1-\delta\left(2 \frac{1-\varepsilon}{1+2 \varepsilon}\right)\right) \frac{1}{2} .
$$

Consequently

$$
\left|z_{n}-\frac{x_{\beta(n)}+x_{\gamma(n)}}{2}\right| \leq\left(1-\delta\left(2 \frac{1-\varepsilon}{1+2 \varepsilon}\right)\right) \frac{1}{2}
$$

As $\delta(\cdot)$ is a continuous function in [0,2[ we have

$$
\left|z_{n}-\frac{x_{\beta(n)}+x_{\gamma(n)}}{2}\right| \leq\left(1-\delta\left(2^{-}\right)\right) \frac{1}{2} \text {. }
$$

Hence

$$
\left|\left[z_{n}\right]\right| \leq \frac{1}{2}\left(1-\delta\left(2^{-}\right)\right)+\frac{1}{2} .
$$

But by hypothesis, it is known that $\varepsilon_{0}(X)<2$ and consequently $\delta\left(2^{-}\right) \neq 0$; therefore we obtain that $R\left(0, M_{\rho}\left(\left(x_{n}\right), \beta, \gamma\right)\right)<1$ which completes the proof.

COROLlary 2.2. Let $X$ be a weakly-orthogonal Banach lattice so that $\varepsilon_{0}(X)<2$. Then $X$ has the AMCP.

PROof. It is known that every weakly-orthogonal Banach lattice is WOTH hence we can use the theorem (2.1).

REMARK 2.3. In [8] is proved that every weakly-orthogonal Banach lattice has the FPP. Using the same technique it is easy to see that ever weaklyorthogonal Banach lattice has the AMCP.

On the other hand when $X$ is a Banach space with a Schauder basis $\left(e_{n}\right)$ we can define the following coefficients associated to $\left(e_{n}\right)$. (See [4].) $c:=\max \left\{\sup \left\{\left\|P_{n}\right\|: n \in \mathbb{N}\right\}, \sup \left\{\left\|I-P_{n}\right\|: n \in \mathbb{N}\right\}\right.$ where $P_{n}$ is the natural projection on the segment $[1, n]\}$. $\eta:=\sup \left\{\left\|I-P_{F}\right\|: F\right.$ is any segment in $\left.N\right\}$.

It is easy to prove that if $X$ is a Banach space with a Schauder basis $\left(e_{n}\right)$ so that $2 c+\eta<4$ then $X$ has the AMCP whenever $X$ is WOTH.

\section{References}

[1] D. Alspach, 'A fixed point free nonexpansive map', Proc. Amer. Soc. 82 (1981), 423-424.

[2] J. M. Borwein and B. Sims, "Nonexpansive mapping on Banach lattices and related topics', Houston J. Math. 10 (1984), 339-356. 
[3] J. Elton, P. K. Lin, E. Odell and S. Szarek, 'Remarks on the fixed point problem for nonexpansive maps', Contemp. Math. 18 (1983), 87-120.

[4] J. Garcia Falset and E. Llorens Fuster, 'A geometric property of Banach spaces related to the fixed point property', J. Math. Anal. Appl. to appear.

[5] L. A. Karlovitz, 'Existence of fixed points for nonexpansive mappings in space without normal structure', Pacific J. Math. 66 (1976), 153-159.

[6] W. A. Kirk, 'A fixed point theorem for mappings which do not increase distance', Amer. Math. Monthly 72 (1965), 1004-1006.

[7] B. Maurey, 'Points fixes des contractions de $L_{1}$ de certaines faiblement compacts', in: Seminar on Functional Analysis, 1980-81 (Exp. No. VIII, École Polytech., Palqiseau, 1981).

[8] B. Sims, 'Orthogonality and fixed points of nonexpansive maps', Proc. Centre Math. Anal. Austral. Nat. Univ. 20 (1988), 178-186.

\author{
Departmento de Análisis Matemático \\ Universidad de Valencia \\ Doctor Miliner 50 46.100 Burjassot \\ Spain
}

